\title{
Tratamento endovascular dos aneurismas da aorta torácica: passo a passo
}

\author{
Eduardo K. SAADI
}

O tratamento cirúrgico dos aneurismas da aorta descendente $(\mathrm{AD})$ tem elevadas taxas de morbidade e mortalidade, mesmo em centros de excelência. $\mathrm{O}$ tratamento endovascular dos aneurismas da aorta torácica visa excluir o aneurisma da circulação. Esta abordagem inovadora no tratamento dos aneurismas da aorta torácica descendente foi desenvolvida em 1994 pelo grupo da Stanford University.

Preliminarmente, os autores procederam o implante percutâneo por via femoral, de stents autoexpansíveis recobertos por Dacron, em 13 casos de aneurismas torácicos crônicos, com diâmetro médio de $6,1 \mathrm{~cm}$. A exclusão imediata foi observada em 12 pacientes, e não houve óbito ou paraplegia na fase hospitalar, nem no seguimento médio de 11,6 meses. Em ensaio clínico desenvolvido subsequentemente, os mesmos autores ampliaram a experiência para 103 implantes, a maioria dos quais com risco cirúrgico proibitivo, e obtiveram sucesso imediato em $83 \%$ dos casos. A mortalidade precoce foi de $9 \%$, paraplegia foi observada em 3\% dos pacientes, e 7\% desenvolveram acidentes isquêmicos cerebrais durante a internação. O seguimento tardio de 3,7 anos demonstrou uma sobrevida livre de eventos de $53 \%$, e o grupo passou a recomendar esta técnica a pacientes selecionados de alto risco operatório [1].

Entretanto, uma experiência maior com a exclusão percutânea de $\mathrm{AD}$ foi posteriormente publicada por Buffolo et al. [2], que entre 1996 e 2002 procederam 191 implantes de endopróteses, em casos de dissecção tipo B $(n=120)$, aneurismas torácicos verdadeiros $(n=61)$, hematomas ou úlceras penetrantes ( $\mathrm{n}=6)$ e lesão traumática da aorta descendente $(n=4)$. Em todos os pacientes, foi utilizada anestesia geral, hipotensão induzida (PAM entre 50 e $60 \mathrm{mmHg}$ ), heparinização apenas durante o procedimento (5000 UI/IV), e stents recobertos por malha de polyester (Braile Biomédica ${ }^{\circledR}$ ), cujo tamanho foi calculado como excedendo em $10 \%$ a $20 \%$ o diâmetro da aorta (oversizing). A taxa de sucesso nos implantes, definida como a obliteração da laceração de íntima ou a completa exclusão dos aneurismas sem extravazamentos, foi de $91 \%$; a mortalidade hospitalar foi de $10 \%$, a conversão para cirurgia necessária em 6 pacientes (3\%), e nenhum caso de paraplegia foi observado [2].

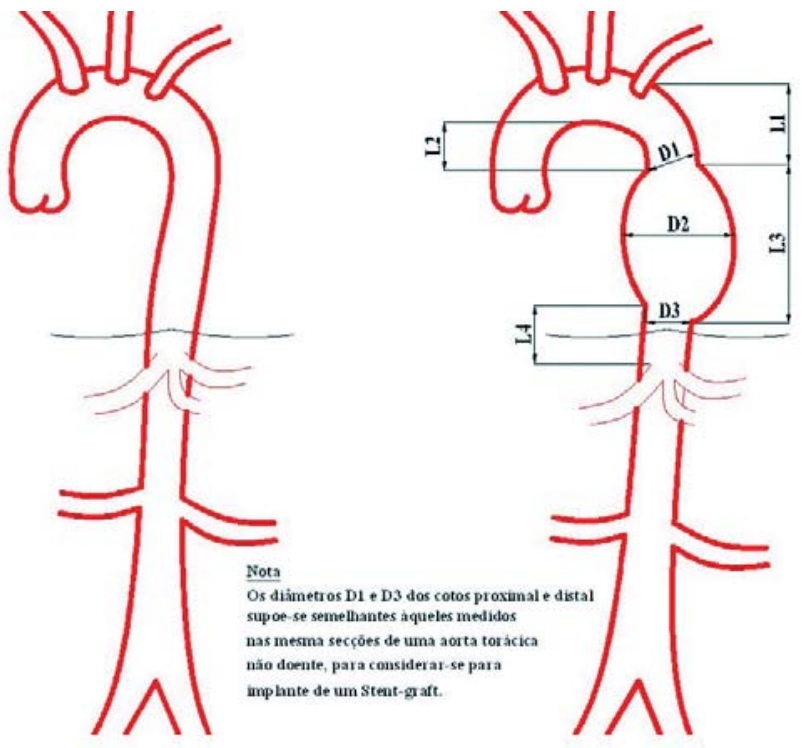

Medidas necessárias para planejamento da correção endovascular de um aneurisma verdadeiro da aorta torácica descendente: onde $D$ corresponde aos diâmetros e $L$ às extensões

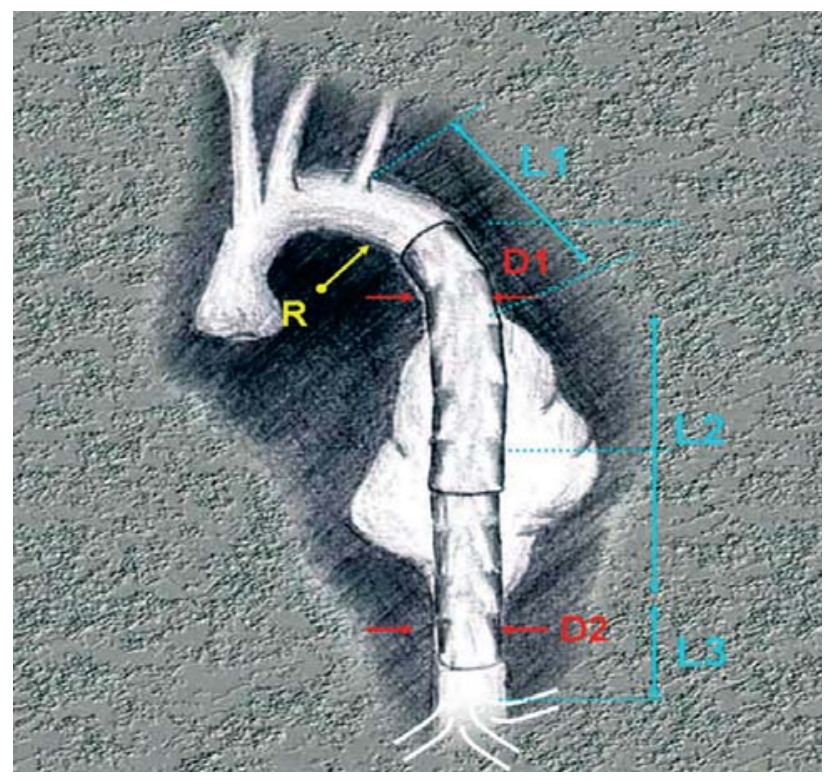

Diâmetros e extensões que devem ser medidos 
Poucos são os estudos prospectivos e randomizados comparando os dois tipos de tratamento. Em um ensaio clínico multicêntrico, os resultados iniciais do tratamento endovascular foram melhores do que os resultados cirúrgicos: mortalidade de $2.1 \%$ (endovascular) versus $11.7 \%(P<.001)$ [cirurgia convencional], mesmo em pacientes de baixo risco [3]. Os resultados foram mantidos em 5 anos de acompanhamento demonstrando que o tratamento endovascular foi superior à cirurgia aberta, com menor mortalidade relacionada ao aneurisma e menor taxas de eventos adversos [4].

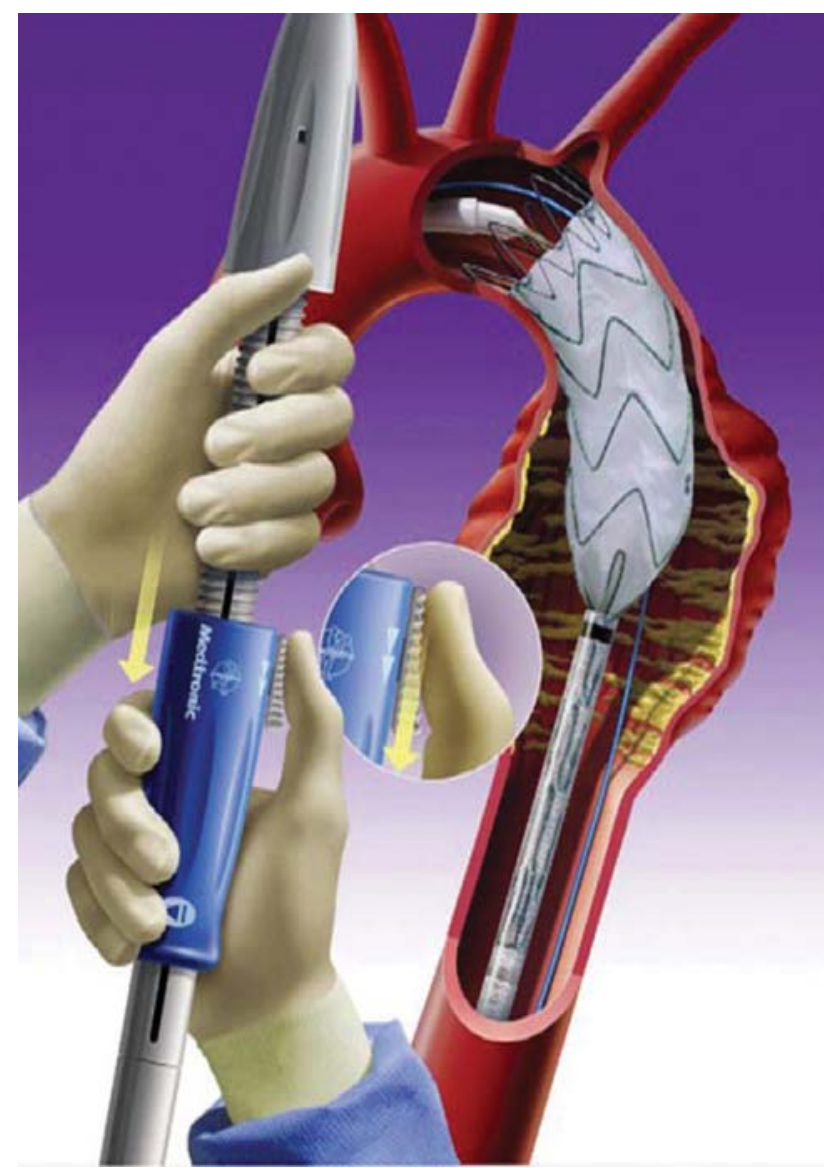

Liberação de uma endoprótese de nitinol com poliéster com “freeflo" junto à subclávia esquerda em aneurisma da aorta torácica descendente

Recentemente, publicamos o resultado de um estudo multicêntrico brasileiro com 255 pacientes que mostrou que a curva de sobrevida em 36, 60 e 112 meses foi 96\%, 89.1\%, $85.1 \%$ e a curva livre de reintervenção para o mesmo período foi de $93.6 \%$, 82.7\%, 57.2\%, respectivamente. O estudo mostrou uma baixa morbi-mortalidade no grupo com diversos tipos de doenças da aorta torácica, mas depois de um período de 42 meses houve um aumento do percentual de novas intervenções [5].

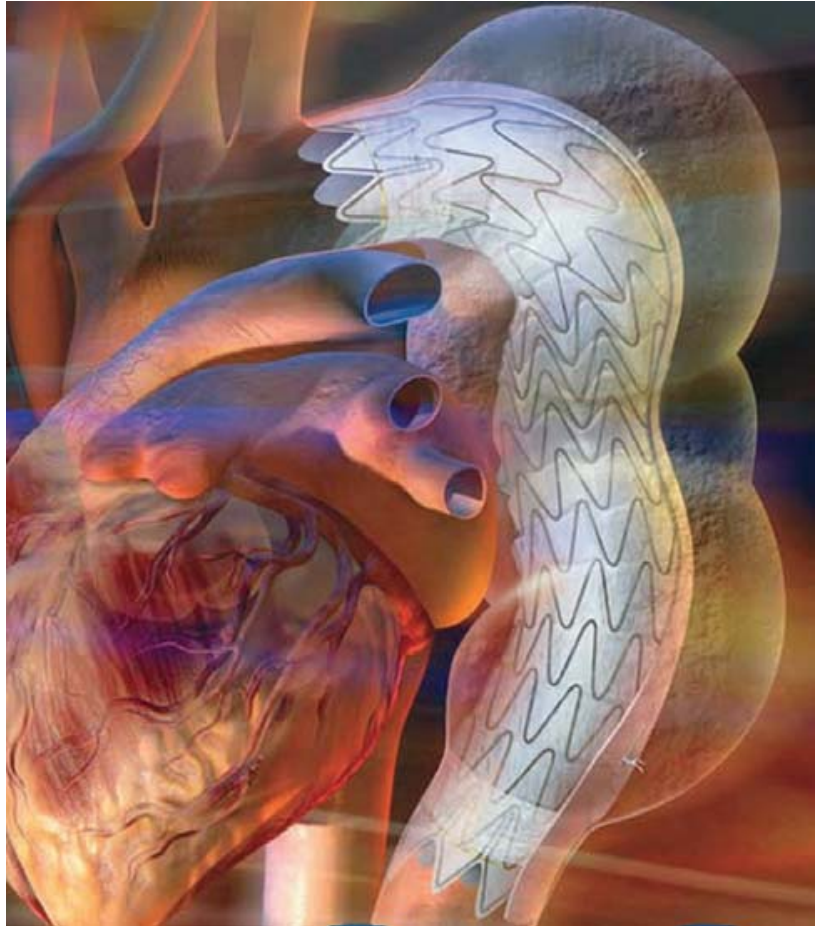

Endoprótese de nitinol com PTFE expandido sem "freeflo" implantada logo após a origem da subclávia esquerda

Inicialmente utilizada somente nos aneurismas da aorta descendente, as indicações da endopróteses foram expandidas para as dissecções, hematomas intramurais, ruptura traumática de aorta, úlcera penetrante, falsos aneurismas, entre outras. As indicações iniciais incluíam uma aorta após a subclávia esquerda de, no máximo 35 mm

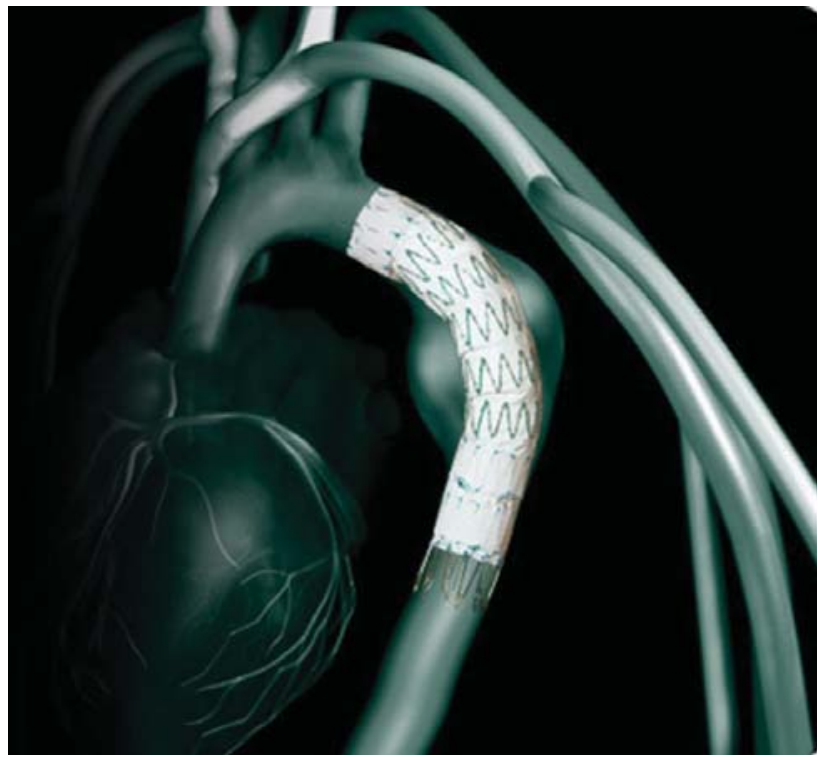

Endoprótese de aço inox com poliéster e "freeflo" distal 


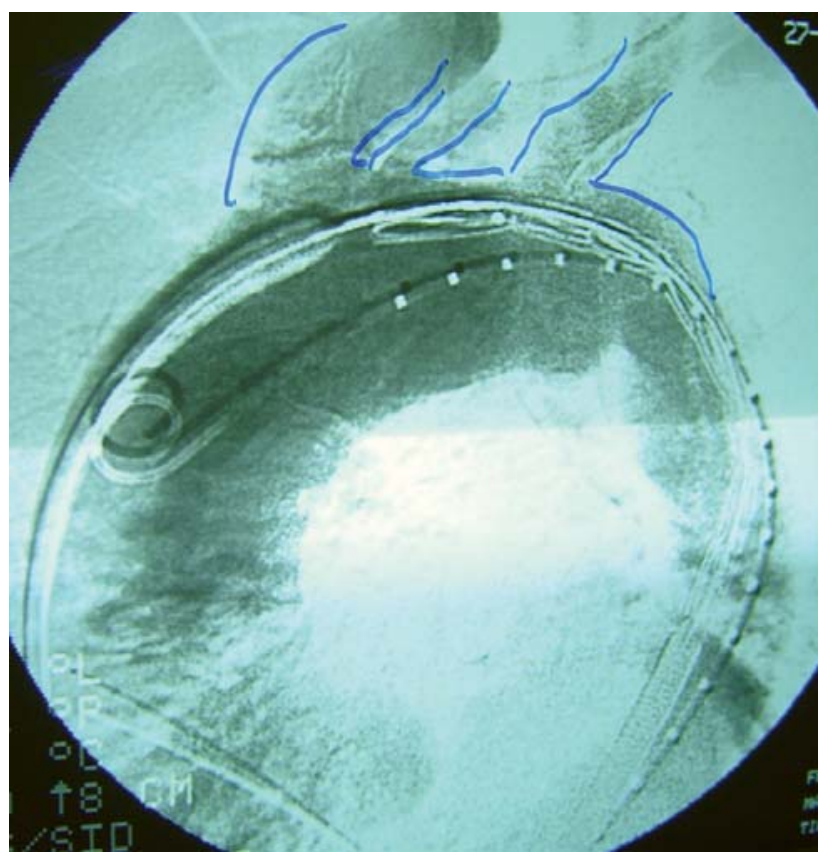

Endoprótese de nitinol com PTFE expandido sem "freeflo" implantada logo após a origem da subclávia esquerda

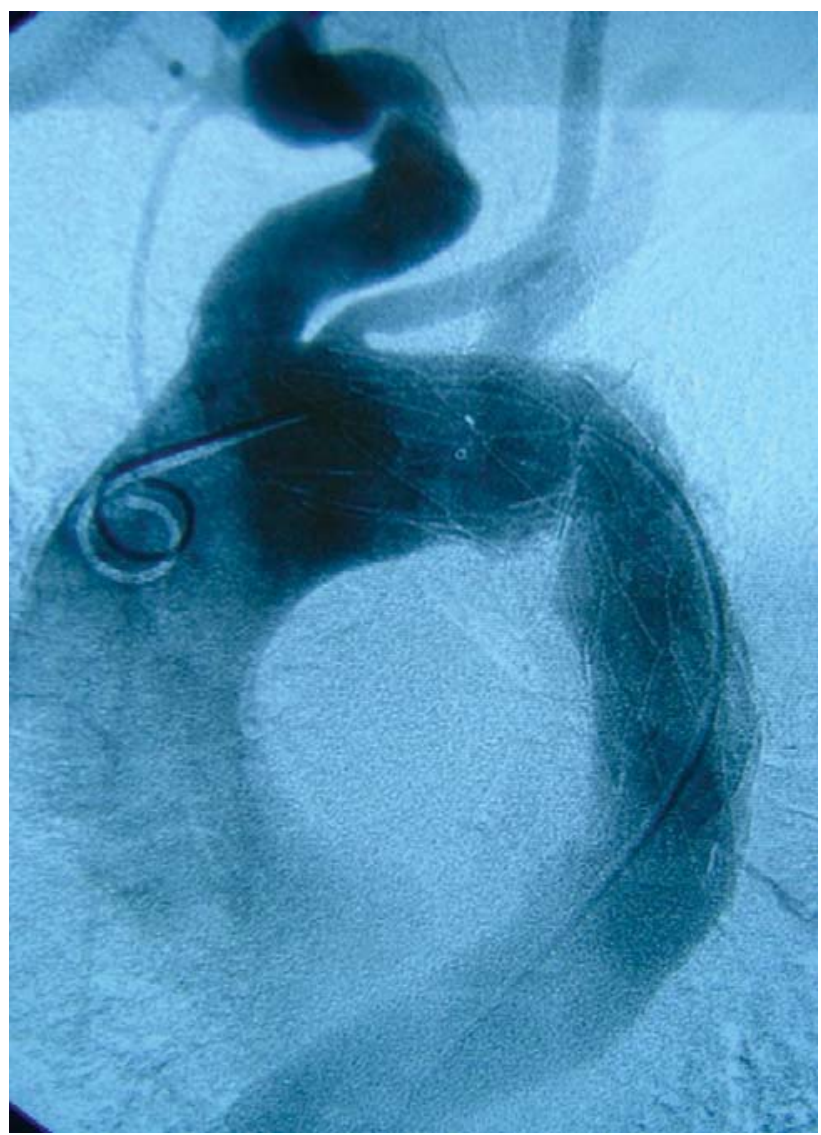

Endoprótese liberada com "freeflo" sobre a subclávia esquerda de diâmetro, um colo entra a subclávia esquerda e o início do aneurisma de $1,5 \mathrm{~cm}$ e um colo distal de, no mínimo, 1 cm. As indicações foram expandidas com a evolução dos materiais e a experiência adquirida. Hoje, em cerca de 60 \% dos casos, a indicação pode ser considerada "off label” e inclui dissecções, colos curtos, correções de aneurismas de arco aórtico com cirurgia híbrida e tóraco-abdominais com procedimentos híbridos e endopróteses ramificadas. A aorta torácica é dividida em zonas, conforme o local onde precisamos ancorar a endoprótese.

Não há dúvidas de que estes resultados representam um importante avanço no tratamento de uma das doenças cardiovasculares de mais alta morbidade e mortalidade, altamente desafiadora para os cirurgiões.

\section{REFERENNCIAS}

1. Dake MD, Miller DC, Mitchell RS, Semba CP, Moore KA, Sakai T. The "first generation" of endovascular stent-grafs for patients with aneurysms of the descending thoracic aorta. J Thorac Cardiovasc Surg. 1998;116(5):689-703.

2. Buffolo E, Fonseca JH, Souza JA, Alves CM. Revolutionary treatment of aneurysms and dissections of descending aorta: the endovascular approach. Ann Thorac Surg. 2002;74(5):S1815-7.

3. Bavaria JE, Appoo JJ, Makaroun MS, Verter J, Yu ZF, Mitchell RS; Gore TAG Investigators. Endovascular stent grafting versus open surgical repair of descending thoracic aortic aneurysms in low-risk patients: a multicenter comparative trial. J Thorac Cardiovasc Surg. 2007;133(2):369-77.

4. Makaroun MS, Dillavou ED, Wheatley GH, Cambria RP; Gore TAG Investigators. Five-year results of endovascular treatment with the Gore TAG device compared with open repair of thoracic aortic aneurysms. J Vasc Surg. 2008;47(5):912-8.

5. Almeida RM, Leal JC, Saadi EK, Braile DM, Rocha AS, Volpiani $\mathrm{G}$, et al. Thoracic endovascular aortic repair: a Brazilian experience in 255 patients over a period of 112 months. Interact Cardiovasc Thorac Surg. 2009;8(5):524-8.

\section{ROTINAS PARAIMPLANTE DE ENDOPRÓTESES AÓRTICAS TORÁCICAS Passo a passo}

\author{
Dr. Eduardo K. Saadi
}

- MONITORIZAÇÃO(ECG+OXIMETRIA+PNI)

- PUNÇAO VENOSA MSE ABOCATH 14 OU 16(anestesista) 
- BLOQUEIO(anestesista)+SEDAÇÃO OU LOCAL + SEDAÇÃO. Geral se eco transesofágico.

- PLACA ELETROCAUTÉRIO, FOCO CIRÚRGICO E ASPIRADOR

- SONDAGEM VESICAL (enfermeira)

- DEGERMAÇÃO MSE E REG INGUINAL BILATERAL PVPI DEGERMANTE

- PVPI TÓPICO + CAMPOS ESTERILIZADOS

- DISSECÇÃO INGUINAL UNILATERAL NO LADO DE MELHOR PULSO E MELHOR ACESSO AVALIADO PRÉ OP PELA TC

- ACESSO ARTERIAL BRAQUIAL ESQ. SN (INTRODUTOR 5F E CATETER PIG LONGO) [RARAMENTE USADO].

- PUNÇÃO FEMORAL CONTRALATERAL E INTRODUTOR 6F

- MATERIAL CIRÚRGICO: 1 BENDEJA VASCUALAR(4 CLAMPES-2 SEMI-RETOS E 2 SATINSKY PEQUENOS,PORTA-AGULHA VASCULAR E PINÇA ANATÔMICA) + 2 BECKMANS GRANDES + 1 BÁSICA +2 FITAS CARDÍACAS + 2 SILASTIC

- INTRODUTOR 7F FEMORAL UNILATERAL GUIA TEFLONADA 0,035J + GUIA HIDROFÍLICA 0,035 260 CM. PIG CENTIMETRADO CONTRALATERAL À INTRODUÇÃO DO CORPO DA PRÓTESE E GUIA EXTRA STIFF LANDERQUIST COOK 260 CM NO LADO DA LIBERAÇAO DA ENDOPRÓTESE.

- AORTOGRAFIA DIGITAL VIA FEMORAL(ou braquial) EM OAE COM VISUALIZAÇAO DESDE À CARÓTIDA ESQ., SUBLÁVIA ESQUERDA ATÉ A PARTE DISTAL DO
ANEURISMA COM PIG TAIL CENTIMETRADO (GRADUADO COM 20 CM DE MARCAS DE OURO)

- CONFIRMAR AS MEDIDAS DA TOMOGRAFIA (DIÂMETRO E EXTENSÃO)

-HEPARINIZAÇÃO SISTÊMICA(1 A 2 MG/KG)

- ESCOLHA E COLOCAÇAO DA PRÓTESE COM ANGIOGRAFIA E REFERÊNCIA ÓSSEA

- LIBERAÇÃO DA ENDOPRÓTESE COM CONTROLE PROXIMAL JUSTA SUBCLÁVIA OU COBRINDO ESTA SE NECESSÁRIO

- RETIRAR CATÉtER CENTIMETRAdO COM GUIA

- balonar para acomodaÇão(PROXiMAL e DISTAL) COM BALÃO CODA/COOK 35 ML

- CONTROLE ANGIOGRÁFICO FINAL EM MODO AORTA DIGITAL

- ARTERIORRAFIA FEMORAL COM PROLENE 5-0

- PROTAMINA

- FECHAMENTO SUBCUTÂNEO VICRIL 2-0 AGULHA GRANDE E PELE MONO 3-0

\section{-AORTOGRAFIA-}

Contraste não iônico puro(Iopamiron) baixa osmolaridade ou diluído 2/3 contr. e 1/3 SF.

Tórax- 18 ou $20 \mathrm{ml} / \mathrm{seg}$. total $40 \mathrm{ml}$ por injeção com bomba. Pressão 1200.

Abdome- $15 \mathrm{ml} / \mathrm{seg}$. total $30 \mathrm{ml}$ por injeção com bomba. Pressão 800. 